\title{
Políticas ciudadanas, inmigración y cultura: El caso del deporte en la ciudad de Barcelona
}

\section{City policies, immigration and culture: Sports in Barcelona}

\author{
Ricardo Sánchez Martín \\ Facultat de Psicologia, \\ Ciènces de l'Educació i de l'Esport Blanquerna \\ Universitat Ramon Llull. Barcelona
}

\section{RESUMEN}

En el Plan Estratégico del deporte de la ciudad de Barcelona se considera la necesidad de facilitar y promover la práctica de la actividad físico-deportiva de toda la población, dadas sus posibilidades como elemento generador de calidad de vida y salud, de participación ciudadana y de integración social. Como resultado de la investigación que se presenta se han determinado las barreras socio-culturales para la práctica de actividades físico-deportivas, se muestran los hábitos de práctica deportiva de los colectivos extranjeros y se establecen los criterios para la política municipal de promoción deportiva de la ciudadanía para el caso concreto de Barcelona. Se trata de una Investigación Social Participativa que incluye análisis documental, estudios cuantitativos, técnicas cualitativas, así como técnicas de investigación colaborativa, sesiones participativas y asambleas.

Palabras clave: Inmigración, Integración, Políticas deportivas, Culturas deportivas, Barreras socioculturales, Promoción deportiva.

\section{SUMMARY}

The Strategic Plan of the city of Barcelona considers the need to both facilitate and promote the practice of sporting activities, since it is proved to enhance people's quality of life and health as well as promote civic participation and social integration. This analysis shows the socio-cultural barriers for the practice of sporting activities and foreign groups' sporting habits as well as the local policy criteria for the promotion of sports among citizens for the specific case of the city of Barcelona. It is a Social Participative Research including a documentary analysis, quantitative studies, qualitative skills as well as cooperative research techniques, participative sessions and meetings.

Key words: Immigration, Integration, Sporting Policies, Sporting Cultures, Socio-cultural Barriers, Sportive Promotion. 


\section{INTRODUCCIÓN}

El texto que presentamos a continuación tiene su origen en una investigación encargada por el Institut Barcelona Esports del Ajuntament de Barcelona ${ }^{1}$. El estudio, titulado Barcelona, Inmigración y Culturas deportivas: estudio para determinar criterios de intervención y promoción deportiva, se realizó durante los años 2006-2008.

La llegada de diversos colectivos de inmigrantes, de procedencias y culturas diferentes, crea un nuevo reto a la política de promoción deportiva existente. Una política pública que pretende hacer que la práctica del deporte y sus beneficios lleguen a todo el mundo sin exclusiones. Por este motivo se planteó desarrollar una investigación participativa para conocer las barreras que encuentran estos colectivos para la práctica deportiva, con el fin de señalar los criterios básicos para lograr una intervención pública más eficaz.

Los objetivos planteados eran:

1. Conocer la realidad del fenómeno migratorio en Barcelona.

2. Analizar las políticas de promoción de la práctica físico-deportiva de los recién llegados.

3. Estudiar la realidad de la práctica deportiva: barreras y demandas.

4. Establecer los criterios para la promoción de la práctica físico-deportiva de los recién llegados.

Es cada vez más habitual encontrarnos con discursos que, desde diferentes ámbitos (administrativos, académicos o sociales), muestran el deporte como un vehículo para la integración o la inclusión social. Por ello se amplían los programas deportivos dirigidos a las poblaciones a las que se considera con un déficit o riesgo de integración social: jóvenes desfavorecidos, mujeres, personas con discapacidad, inmigrantes. Más allá de planteamientos simplistas que esperamos denunciar a lo largo del texto, lo cierto es que el deporte se ha convertido en un hecho significativo de alcance global.

Teóricamente partimos de un posicionamiento que hace del deporte, como hecho social total, un potente mecanismo ritual para la reproducción de la sociedad (Sánchez 2003). Especialmente significativo para una sociedad cuyo futuro se problematiza desde diferentes corrientes teóricas, políticas y sociales: neoliberales hiperindividualistas que abanderan el interés particular en clave hegemónica y como motor de cambio social; posmodernis-

\footnotetext{
${ }^{1}$ La investigación se realizó junto con el Dr. Jaume Bantulà de la Facultat de Psicologia, Ciències de l'Educació i de l'Esport Blanquerna de la Universitat Ramon Llull.
} 
tas que anuncian la fragmentación y desaparición de la sociedad como proyecto; o teóricos de la globalización que consideran que la actual sociedad no sobrevivirá al debilitamiento del Estado-Nación (Outhwaite 2008).

Como semántica ritualizada, el sistema deportivo actual contiene, expresa y configura las características de la sociedad en la que se produce y, como consecuencia de ello, se pluraliza ante la creciente complejidad de la sociedad contemporánea (posmoderna, líquida, del riesgo, hipermoderna, etc.) (Sánchez 2004). En este escenario complejo, el sistema deportivo, tanto formal como informal, presenta unos elementos muy especiales que le convierten en un instrumento clave en los procesos de integración y reproducción social. Ya señalábamos en otro lugar (Sánchez 2009)² que la fuerza del deporte en la sociedad actual deviene de ser tanto un productor de sentido comunicacional y discursivo, como de sentido práctico incorporado; sustentado por una ética basada en el sentir en común (estética) y, en última instancia, por su capacidad de producir capital social.

En efecto, el deporte produce sentido porque funciona como un discurso redundante que reduce la complejidad de las metanarrativas sobre lo social y posibilita la producción selectiva de las formas psíquicas y sociales. El deporte genera "sentido práctico" (Bourdieu 1997). Un conocimiento aplicado que no necesita de la reflexión teórica y que no se expresa solo discursivamente. Un sentido incorporado, hecho cuerpo, que hace de la corporalidad un instrumento comunicativo de gran relevancia.

Esta corporalidad es un factor clave en la producción de una razón sensible (Maffesoli 1997) que, más allá de la razón instrumental, puede generar una razón estética y permitir la reunión de la sociedad —el sistema, la estructura, lo racional- con la socialidad — la voluntad estética del estar juntos-. Un sentir en común que se convierte en el cemento de la sociedad.

Por todo ello, las diversas prácticas deportivas (el sistema de los deportes) permiten también la expresión y configuración de diferenciados y distintivos habitus (Bourdieu 1997). Dibujan estilos de vida, estructuras variadas de percepción, interpretación y acción social que, más allá del deporte, pueden ayudar a cambiar la estructura de capitales sociales y la producción de capital simbólico y, por tanto, a su reubicación en el sistema de las posiciones sociales.

Por último, las actividades físico-deportivas pueden contribuir al incremento de las relaciones sociales y a una ampliación y densificación de las redes sociales que están en la base de la creación de capital social (Maza 2006).

\footnotetext{
2 Texto editado de la conferencia impartida en la Alta Scuola di Psicologia de la Università Cattolica del Sacro Cuore de Milano en Noviembre de 2008 (Sánchez 2009).
} 
Metodológicamente, partimos de una epistemología del conocimiento fundamentada en la comunicación y la simetría entre investigadores e investigados (Domínguez y Coco 2000). Una tarea de investigación que se acerca al problema no sólo para conocer la realidad, sino para transformarla desde el conocimiento y la cooperación; que está, por tanto, orientada a la acción y resolución de problemas sociales e implica, como señala Buxó (2002) para el caso de la antropología, un requerimiento de participación activa acentuando el consenso, el consentimiento, la cogeneración de datos y la codefinición de problemas, de forma tal que se investiga para transformar y democratizar (Alberich 2000).

Por ello, priorizamos el pluralismo metodológico a través de un diseño multimétodo que nos permitiese profundizar en el objeto de estudio (Domínguez y Coco 2000). Se trata de una estrategia de triangulación de diferentes técnicas de observación y de análisis para el estudio de un mismo aspecto de la realidad sociodeportiva, algo, por otra parte, nada ajeno a la propia tradición etnográfica. Como técnicas de investigación se utilizaron:

A) Observación: Búsqueda documental y estadística; observación directa; entrevistas semiestructuradas en profundidad; entrevistas en grupo; Método Delphi (Brunet, Pastor y Belzunegui 2002; Martín 2001).

B) Participación social: Dinámica de grupos; asambleas (Villasante, Montañés y Martí 2000, 2001; Gabarrón y Hernández 1994).

C) Análisis: Análisis documental, estadístico y de contenido.

LA POBLACIÓN EXTRANJERA EN BARCELONA: POLÍTICAS ADMINISTRATIVAS Y DEPORTE

El informe estadístico del Ajuntament de Barcelona (2006a) sobre la evolución demográfica de la población extranjera durante el 2005 presenta los siguientes datos:

El número de extranjeros empadronados representa un 15,9\% sobre el total de población de la ciudad. Los extranjeros comunitarios son el único grupo que durante 2005 aumentó su tasa de crecimiento respecto al año anterior. Por nacionalidades, el primer país de origen es Ecuador (12\% del total de extranjeros), seguido de Perú, Marruecos, Colombia, Italia, Pakistán y Argentina. Del total de extranjeros residentes en la ciudad, el $90 \%$ se ha dado de alta en el padrón municipal hace menos de 5 años. En cuanto a su perfil, el informe señala que los extranjeros son en su mayoría hombres (52\%), especialmente los procedentes de Asia Central (75\%), África subsahariana (68\%), África del Norte y Magreb (66\%), y Oriente Medio (63\%). Por grupos de edad, la mitad pertenecen al grupo de 25 a 39 años. Por nivel de estudios, casi la mitad ha realizado estudios primarios, mientras el $28 \%$ tiene estudios superiores. 
Casi la mitad de los extranjeros residen en los distritos de l'Eixample, Ciutat Vella y Sants-Montjuïc. L'Eixample, con un 17\% del total de extranjeros, ha superado a Ciutat Vella, con un 15,7\%; sin embargo, Ciutat Vella mantiene un porcentaje mayor de foráneos con relación al total de los vecinos (36\%). Los extranjeros procedentes de Asia Central, Sudeste Asiático, África del Norte y Magreb residen mayoritariamente en Ciutat Vella (más del $30 \%$ ), mientras que los de Oriente Medio y Unión Europea lo hacen en l'Eixample (20\%) y los del África subsahariana en Nou Barris (27\%). El resto de grupos se distribuyen más proporcionadamente entre diferentes distritos. Como se puede comprobar, la población extranjera en Barcelona no se reparte de forma homogénea por todos los distritos. Tampoco existe una distribución paritaria entre géneros, edades y perfiles, por lo que un elemento primordial sería establecer programas adecuados a las necesidades concretas de cada espacio urbano.

Pasamos a explicar las políticas municipales referidas al área de deportes y que se vinculan con el objetivo estratégico del Plan Municipal de Inmigración (PMI), que pretende desarrollar programas adecuados para colectivos en riesgo de exclusión social. Las propuestas planteadas son las siguientes:

1. Ampliar los horarios de las instalaciones deportivas para que los colectivos de inmigrantes tengan un acceso mejor y más flexible.

2. Organizar campeonatos con grupos de inmigrantes que puedan competir con otros equipos, tal como los que organiza la asociación IbnBatuta.

3. Hacer labor de divulgación de los servicios y equipamientos que ofrece la institución entre los recién llegados. Informar de la posibilidad de acceder y de cómo hacerlo, como individuo y como equipo.

4. Facilitar espacios para que los diferentes colectivos puedan practicar deportes, teatro y danza.

5. Llevar a cabo un seguimiento de la normativa de las federaciones deportivas, para comprobar las posibilidades de normalizar la participación de los inmigrantes no nacionalizados en las competiciones organizadas por aquéllas, puesto que se establecen límites irregulares en estas normativas para los menores de dieciocho años.

6. Organización de torneos de mutuo reconocimiento en los deportes habituales: fútbol, tenis mesa y balonmano, entre deportistas autóctonos y la población inmigrante.

7. Organización de festivales de exhibición de los deportes tradicionales de los países de origen de los inmigrantes residentes en Barcelona: críquet (paquistanís), palín (chilenos), etc.

8. Establecer zonas deportivas gratuitas en cada barrio para jóvenes. 
Ramón Sanahuja, del Gabinete Técnico de Inmigración del Ayuntamiento, subrayó la conveniencia de tratar el deporte bajo la premisa de la normalización, indicando que no existía una política específica para deporte e inmigración, "en todo caso — añadió- se puede hablar de diferentes actuaciones en función de los Planes Territoriales de Inmigración para los Dis-

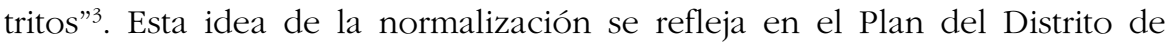
Sants-Montjuïc que se conoce como "Pla per a la promoció de la convivència i el civisme" (2006-2007). Según consta en el plan, para lograr la normalización del hecho migratorio y la diversidad cultural, se deberían tener en cuenta, entre otros, los siguientes aspectos: a) Evitar organizar servicios específicos dirigidos a la población inmigrada más allá de los estrictamente necesarios (jurídicos, capacitación lingüística). b) Evitar una actuación meramente asistencial y compensatoria hacia el hecho migratorio que refuerce actitudes de dependencia y agravios comparativos del resto de población. En el plan se enfatiza la necesidad de establecer un discurso político y técnico mínimamente cohesionado y propone que se fundamente en cuatro argumentos:

a) Uso de términos como ciudadanía y vecinos en vez de inmigrantes y autóctonos.

b) Convivencia basada en una ciudadanía de derechos y deberes para todo el mundo, lo cual implica la participación de los nuevos vecinos y vecinas en las dinámicas sociales, políticas y económicas del Distrito.

c) Aceptar que la diversidad es un factor clave para entender y actuar en sociedad, que hay muchos elementos de diversidad (edad, género, origen, cultura, religión, clase social...) y que hace falta reconocerla como un potencial enriquecedor y no como un obstáculo para la cohesión social.

d) La diferencia no supone desigualdad, la lucha contra las desigualdades socio-económicas es una de las prioridades de actuación.

Aunque ninguna de estas propuestas hace referencia directa a la actividad física y el deporte, sí que recogen el planteamiento teórico sobre el que fundar un programa de promoción deportiva adaptado al plan del distrito. Sin embargo, observaremos más adelante, dentro del debate teórico, la paradoja de alguna de estas propuestas.

\footnotetext{
${ }^{3}$ Entrevista realizada el 20 de abril de 2007.
} 
El DePORTE EN LA CIUDAd DE BARCELONA: HÁBitos, BARRERAS Y DEMANDAS DE LA POBLACIÓN EXTRANJERA

Dentro de los estereotipos habituales sobre la población extranjera con relación al deporte se afirma que este colectivo tiene una baja tasa de práctica deportiva en relación a la del total de la población por motivos de origen étnico y cultural. Así, en la encuesta de hábitos deportivos de los escolares de la ciudad de Barcelona podemos ver que en Ciutat Vella la práctica deportiva fuera del horario escolar es de un 43,5\% cuando en el resto de distritos supera el 60\%. Este hecho se interpreta como sigue:

Se establece, por lo tanto, una correlación directa entre los niveles de práctica deportiva fuera del horario lectivo entre los escolares de la ciudad y el origen y procedencia de los mismos [...] Los niños y niñas inmigrantes tienden a tener un índice de práctica deportiva inferior (Ajuntament de Barcelona 2007: 14).

Sin duda, en este debate no se han incorporado otros diacríticos que, con mayor peso, condicionan la práctica deportiva y que sí se tienen en cuenta desde hace décadas con respecto a la población en general. Y es que el marco que posibilita la práctica deportiva es multifactorial y en él intervienen elementos como la edad, el género, el nivel de estudios, la actividad profesional, el estado civil, la posición económica, etc.

Análisis cuantitativo de los hábitos deportivos de los ciudadanos barceloneses a partir del diacrítico origen de nacimiento

Para el presente análisis cuantitativo se parte de la base de datos que proporciona la Enquesta. Hàbits Esportius a Barcelona. Presentació de resultats, del Ajuntament de Barcelona (2006b). Los análisis estadísticos que se han realizado entre las diferentes variables son:

1. Estudio de las correlaciones a través de tablas de contingencia.

2. Método de las diferencias entre proporciones (Puntuación Z):

Este análisis estadístico se ha realizado mediante el Método de las diferencias entre proporciones $(Z)$, utilizando un coeficiente de significación de $\alpha=0,05$, con la finalidad de determinar si existen diferencias estadísticamente significativas entre las proporciones establecidas para cada una de las variables y sus valores en relación a las diferentes categorías estudiadas. Los índices de significación son:

- $Z_{\alpha}$ menor de 1,96. No hay nivel significativo.

- $Z_{\alpha}$ entre 1,96 y 2,57, nivel significativo.

- $Z_{\alpha}$ igual o mayor de 2,58, nivel muy significativo. 
Dada la limitación de espacio de que disponemos para la presentación del trabajo, hemos optado por estudiar alguna de las variables que han sido objeto de atención en otros estudios de carácter más cualitativo. Las categorías utilizadas son las que se han implementado en la encuesta de hábitos deportivos de los barceloneses ya referida.

En cuanto a la práctica deportiva, podemos decir que del estudio de la tabla de contingencia entre origen y práctica deportiva se desprende que los ciudadanos barceloneses con origen en el resto de Cataluña tienen, con un $70,6 \%$, un porcentaje de práctica deportiva superior al resto de grupos, incluido el de los nacidos en Barcelona ciudad (64,9\%), el de los nacidos en el resto de España (64\%), y el de los nacidos en el resto del mundo, que tiene el porcentaje más bajo con un 61, $1 \%$. Aplicando el análisis de puntuación $Z_{\alpha}$, ante la respuesta "Sí practican", la diferencia entre proporciones es muy significativa $\left(Z_{\alpha}=2.98\right)$ cuando analizamos las proporciones de practicantes entre los grupos resto de Cataluña y resto del Mundo a favor del primer grupo. Y resulta significativa $\left(Z_{\alpha}=2,15\right)$ cuando comparamos resto de Cataluña con resto de España $\left(Z_{\alpha}=2,15\right)$, e, incluso, con nacidos en Barcelona ciudad $\left(Z_{\alpha}=-\right.$ 2,04). Sin embargo, no hay que olvidar que otros diacríticos sociales, como por ejemplo el nivel de estudios, establecen diferencias mayores entre grupos. Los ciudadanos con menos que estudios primarios tienen un 45,2\% de práctica deportiva, mientras que los ciudadanos con estudios Universitarios llegan a un 74,6\%.

No obstante, estas diferencias en la práctica deportiva no responden a un menor interés por el deporte. Sumando las respuestas "mucho" y "bastante" interés por el deporte encontramos que los nacidos en el resto del Mundo mantienen un interés por el deporte considerablemente mayor $\left(Z_{\alpha}=-3,66\right)$ que los nacidos en el resto de España. También es significativa la diferencia con los nacidos en la propia ciudad $\left(Z_{\alpha}=-2,44\right)$. Además, sumando las respuestas "poco" y "nada", vemos que hay más ciudadanos nacidos en Barcelona ciudad no interesados por el deporte que ciudadanos nacidos en el resto del mundo $\left(Z_{\alpha}=2,42\right)$. Este menor interés es aún más significativo $\left(Z_{\alpha}=3,66\right)$ en las diferencias entre los nacidos en el resto del Mundo y el caso de los nacidos en el resto de España.

También se encuentran diferencias en la frecuencia de práctica deportiva. En la práctica físico-deportiva de fin de semana es donde encontramos las diferencias más significativas siempre favorables, en cuanto a volumen de práctica, especialmente al colectivo de los nacidos en el resto del Mundo, también, aunque en menor medida, a los nacidos en el resto de la Unión Europea. Los nacidos en el resto del Mundo mantienen diferencias muy significativas con los nacidos en la ciudad de Barcelona $\left(Z_{\alpha}=-6,67\right)$, con los 
nacidos en el resto de España $\left(Z_{\alpha}=-6,18\right)$ y con los nacidos en el resto de Cataluña $\left(Z_{\alpha}=-4,18\right)$.

De entre los motivos para la no práctica, podemos observar que en los porcentajes de los diferentes motivos para la no práctica de los grupos por origen de nacimiento, el motivo "No me gusta el deporte" es aducido por el 17,3\% de los nacidos en el resto de Cataluña y el 15,9\% de los nacidos en Barcelona. Sin embargo, los nacidos en el resto del Mundo sólo lo indican en un 4,3\%. El motivo "Por motivos de salud" que llega al 18,3\% en el caso del colectivo resto de España, sólo llega al 6,5\% en el colectivo resto del Mundo. Este último grupo es el que mantiene porcentajes más altos en los motivos "No tengo tiempo" (73,5\%), "Por falta de instalaciones deportivas" (5\%), "No me enseñaron en la escuela" (1,4\%). En el estudio de proporciones, es el motivo "Por falta de tiempo" donde se encuentran las diferencias más considerables entre los diferentes grupos. Este es el motivo más significativo estadísticamente de entre los aducidos por el colectivo del resto de Mundo con respecto al resto de España $\left(Z_{\alpha}=-7,53\right)$, Barcelona $\left(Z_{\alpha}=-5,53\right)$ y resto de Cataluña $\left(Z_{\alpha}=-4,09\right)$. También es muy ilustrativa $\left(Z_{\alpha}=3,41\right)$ la diferencia entre los ciudadanos nacidos en Barcelona y en el resto de España a favor de los primeros. El motivo "Por falta de instalaciones", es más señalado por el colectivo resto del Mundo. Destaca como diferencia muy significativa $\left(Z_{\alpha}=-3,48\right)$ la que se establece entre este colectivo y los nacidos en Barcelona. Y como diferencia significativa $\left(Z_{\alpha}=-2,27\right)$ la que aparece entre el resto del Mundo y resto de España.

Entre los motivos para la práctica deportiva destaca la respuesta "Para mantenerse o mejorar la salud". Éste es el motivo que establece diferencias más notables, en su detrimento, entre los nacidos en el resto del Mundo y los ciudadanos de Barcelona nacidos en la propia ciudad $\left(Z_{\alpha}=3,49\right)$, los nacidos en el resto de Cataluña $\left(Z_{\alpha}=3,41\right)$ y los nacidos en el resto de España $\left(Z_{\alpha}=6,88\right)$. También encontramos diferencias muy significativas entre los nacidos en el resto de España y los nacidos en Barcelona $\left(Z_{\alpha}=-5,18\right)$ y entre los nacidos en el resto de España y los nacidos en el resto de la UE $\left(Z_{\alpha}=3,57\right)$. Siempre a favor del resto de España.

En la forma de practicar deporte atendiendo al origen de los encuestados se observan, entre otras, las siguientes diferencias porcentuales: En la respuesta "Practica deporte solo, individualmente" los porcentajes más elevados corresponden a UE $(67,1 \%)$, resto de España $(66,2 \%)$, Barcelona ciudad (59,8\%), resto de Cataluña (54,5\%) y resto del Mundo (54,1\%). En la respuesta "Con amigos o compañeros" los porcentajes más elevados corresponden al resto del Mundo (33,3\%), resto de Cataluña (29,4\%), Barcelona ciudad $(27 \%)$, UE $(25,6 \%)$ y resto de España (20,6\%). En la respuesta "Con algún miembro de la familia" los porcentajes en orden decreciente son res- 
to de España (10,8\%), resto del Mundo (10,3\%), Barcelona (10,1\%), resto de Cataluña (9,5\%) y UE (6,1\%). En la respuesta "Como actividad de un club, asociación o federación" los porcentajes, de mayor a menor, son resto de Cataluña $(3,9 \%)$, Barcelona (2\%), resto del Mundo (1,1\%), resto de España $(0,4 \%)$ y UE (0\%).

En cuanto al lugar de práctica deportiva, en la respuesta "Habitualmente" en los "Parques de la ciudad", encontramos que el colectivo de los nacidos en el resto del Mundo establece, a su favor, diferencias muy significativas con los demás: con los nacidos en Barcelona ciudad, $Z_{\alpha}=-8,40$; en el resto de Cataluña, $Z_{\alpha}=-4,87$; en el resto de España, $Z_{\alpha}=-3,59$; y con los nacidos en UE, $Z_{\alpha}=-3,28$. Por el contrario, en la respuesta "Nunca utilizo la montaña para realizar actividades deportivas" encontramos las siguientes diferencias muy significativas estadísticamente entre el grupo de los nacidos en el resto del Mundo y los nacidos en Barcelona $\left(Z_{\alpha}=-4,16\right)$; los nacidos en resto de Cataluña $\left(Z_{\alpha}=-5,15\right)$ y resto de España $\left(Z_{\alpha}=-3,12\right)$.

Por lo que se refiere a la pertenencia a clubes deportivos, encontramos diferencias significativas y muy significativas estadísticamente entre los diferentes colectivos encuestados por origen de nacimiento. Así, tenemos que con menor pertenencia a clubes, los ciudadanos nacidos en el resto del Mundo mantienen diferencias muy significativas con: nacidos en Barcelona ciudad $\left(Z_{\alpha}=8,42\right)$, nacidos en resto de Cataluña $\left(Z_{\alpha}=7,18\right)$, nacidos en resto de España $\left(Z_{\alpha}=2,89\right)$. También tienen menor pertenencia a clubes que los nacidos en resto UE, pero únicamente con diferencias significativas $\left(Z_{\alpha}=2,05\right)$.

Entre los motivos para la no pertenencia encontramos que la respuesta "Encuentro cara la cuota" marca diferencias significativas estadísticamente, en mayor medida de respuestas, entre los nacidos en resto del Mundo y los nacidos en Barcelona ciudad $\left(Z_{\alpha}=-2,25\right)$. También con los nacidos en resto de España $\left(Z_{\alpha}=-2,25\right)$, y, por último, con los nacidos en UE $\left(Z_{\alpha}=-2,11\right)$. No así, la respuesta "No me gusta estar asociado" que marca diferencias muy significativas estadísticamente, en menor medida, entre los nacidos en resto del Mundo y los nacidos en Barcelona ciudad $\left(Z_{\alpha}=3,05\right)$ y, especialmente, con los nacidos en UE $\left(Z_{\alpha}=4,05\right)$; o la respuesta "Es molesto por las obligaciones que implica" que encuentra diferencias muy significativas entre los colectivos de nacidos en $\mathrm{UE}\left(Z_{\alpha}=2,93\right)$, en resto de España $\left(Z_{\alpha}=2,69\right)$ y en Barcelona ciudad $\left(Z_{\alpha}=2,63\right)$, en relación a los nacidos en resto del Mundo, que la han respondido en menor medida.

Para finalizar, comentaremos las opiniones de los diferentes colectivos de ciudadanos barceloneses con respecto a las políticas de promoción deportiva del Ajuntament de Barcelona. Sorprenden especialmente las valoraciones en torno a dos respuestas. En la petición de una política deportiva 
municipal "A favor de mejorar los equipamientos deportivos en la calle" las diferencias significativas y muy significativas estadísticamente se establecen entre el colectivo de los nacidos en Barcelona ciudad con todos los demás grupos, a favor del primero. En efecto, encontramos diferencias muy significativas con respecto a los nacidos en resto de España $\left(Z_{\alpha}=2,96\right)$ y resto de Cataluña $\left(Z_{\alpha}=2,86\right)$. En el análisis también encontramos diferencias significativas del mismo grupo de ciudadanos nacidos en Barcelona ciudad con respecto a los nacidos en resto del Mundo $\left(Z_{\alpha}=2,54\right)$ y $\mathrm{UE}\left(Z_{\alpha}=2,43\right.$. En la respuesta "A favor de que se promueva la marca Barcelona como ciudad del deporte" dentro de las políticas deportivas municipales de la ciudad encontramos diferencias muy significativas estadísticamente a favor del colectivo de los nacidos en resto del Mundo con respecto a: nacidos en UE $\left(Z_{\alpha}=-3,17\right)$, nacidos en resto de Cataluña $\left(Z_{\alpha}=-2,75\right)$ y nacidos en resto de España $\left(Z_{\alpha}=-2,63\right)$. También existen diferencias significativas a favor de los nacidos en Barcelona ciudad con respecto a los nacidos en UE $\left(Z_{\alpha}=2,33\right)$.

\section{Análisis cualitativo}

A partir del análisis documental, de las entrevistas abiertas, del Método Delphi y de las sesiones participativas realizadas con asociaciones y con técnicos municipales ${ }^{4}$, resultan una serie de características básicas de la actividad deportiva de la población extranjera en Barcelona.

Se trata de una práctica deportiva informal donde la diversión es uno de los motivos principales. El espacio utilizado es habitualmente el espacio público. La práctica deportiva mayoritariamente se realiza por propia cuenta, dejando en segundo término los clubes y asociaciones deportivas. Esto no es óbice para que se de una gran importancia a las relaciones sociales (se realiza con amigos, familiares, etc.). El deporte refuerza la identidad cultural y nacional, y su diversidad de actividades y formas de prácticas encuentra a veces un referente en sus deportes específicos. Hay claras diferencias por género y edad, por ejemplo, los hombres jóvenes tienden a

\footnotetext{
${ }^{4}$ Las sesiones participativas se realizaron durante el mes de marzo de 2008 coincidiendo con la Exposición Esport i construcción de ciutadania que se celebró en el Museu Olimpic de la ciudad de Barcelona del 17 al 31 de marzo de 2008. En las dos sesiones participativas que se celebraron, 28 de febrero y 27 de marzo respectivamente, se presentaron datos y reflexiones preliminares que habían surgido durante las diferentes fases de la investigación y se abrió el debate a la aportación de los asistentes, en su mayor parte técnicos municipales, representantes de asociaciones de inmigrantes y asociaciones socioculturales en general. Posteriormente, el 31 de marzo, se realizó la jornada Esport i Immigració con participación activa de los diferentes actores sociales.
} 
practicar deportes de equipo como el fútbol. En cambio, las mujeres, en general, prefieren practicar las gimnasias suaves y las chicas jóvenes eligen la danza, el funky o el hip hop. Ello no impide la práctica del fútbol, especialmente entre los colectivos de mujeres latinoamericanas.

Algunas de estas características, especialmente su práctica informal, el uso del espacio público y el realizarse al margen de las instituciones deportivas, se han tratado como un hecho diferenciador con respecto a la práctica deportiva de la población barcelonesa en general. Nada más lejos de la realidad. Presentamos a continuación, de forma impresionistas, alguno de los rasgos que dibujan las tendencias en las prácticas deportivas de los barceloneses ${ }^{5}$ :

El 67,3\% practica deporte de forma individual, más que en 1999 (52,2\%). La práctica con amigos ha descendido a un 53,6\% (1999, 56,4\%). Como actividad perteneciente a un club, asociación o federación baja al 5,7\% (1999, 10,9\%). Únicamente un 10,1\% de los deportistas están federados. No pertenecen a ningún club o asociación el 76,9\%. El 42,6\% no utiliza nunca instalaciones deportivas para su práctica. Entre los que llevan a cabo actividad física de forma regular este porcentaje aumenta al 92,5\%. Utilizan la calle el 39\%; los parques el 22,8\%; la playa el 9,9\%; y el puerto y/o mar el 5\%.

Las diferencias principales se presentan en los motivos para la práctica y en la forma de llevarla a cabo. Aunque tanto para la población en general como para la de origen extranjero destaca como motivo principal "Por hacer ejercicio físico y mantenerse en forma”, adquiere más relevancia para la población extranjera el motivo "Por diversión y pasar el tiempo". En cuanto a la forma de practicarlo encontramos que en la población en general destaca la realización de deporte de forma individual, mientras que en la población extranjera disminuye este porcentaje y aumenta el de los que prefieren practicarlo en grupo.

Se podría pensar que la sociedad barcelonesa otorga al deporte un mayor sentido de salud personal y cuidado físico que la población inmigrante, ya que ésta lo utiliza más para relacionarse y divertirse. Éste es un argumento habitualmente presente en las entrevistas que hemos realizado. Sin embargo, nuestro análisis cuantitativo matiza esta diferencia. En efecto, parece que los procesos de individualización que caracterizan la modernidad líquida (Bauman 2000) son próximos a las prácticas deportivas reales, pero, sobre todo, están más presentes en el discurso de la población autóctona que en el de los colectivos extranjeros.

\footnotetext{
${ }^{5}$ Con la finalidad de dar una cierta perspectiva diacrónica, presentamos estos datos con relación a los de la encuesta de hábitos deportivos de los barceloneses de 1999.
} 
Las barreras principales para la práctica deportiva que declara la población extranjera son:

a) Económicas: Coste de la instalación, cuota del club o entidad, coste de las fichas federativas, ausencia de equipamientos abiertos, controlados y gratuitos.

b) Sociales: Distancia a las instalaciones deportivas, falta de información, ausencia de espacios públicos adaptados a la diversidad de las prácticas físico-deportivas, mayor número de alternativas de ocio sedentario, falta de tiempo, el género y la nacionalidad, la condición social, falta de educadores-entrenadores.

c) Culturales: Autoexclusión, diferencias culturales en cuando a la salud, uso del tiempo libre, la cultura del cuerpo, diferente valoración de la actividad físico-deportiva. Reducida oferta de prácticas deportivas (mayoritariamente se ofrece fútbol y baloncesto).

d) Institucionales: Dificultades en el acceso al sistema federativo, problemas en el acceso a instalaciones públicas, legislación restrictiva.

Ante estas barreras, estos colectivos, establecen una serie de demandas:

a) Económicas: Gratuidad de los equipamientos deportivos o precios asequibles, ayudas para la reducción de cuotas del club o entidad, reducción del coste de las fichas federativas, equipamientos abiertos, controlados y gratuitos.

b) Sociales: Mejora de la información, adaptación de espacios públicos a la diversidad de prácticas físico-deportivas, educación para el ocio, ampliación en la oferta de espacios de uso deportivo, diversificación de la oferta en función del género, edad, nacionalidad, condición social, formación de educadores-entrenadores.

c) Culturales: Campañas educativas que permitan valorar positivamente la actividad físico-deportiva como calidad de vida. Difusión de las prácticas multiculturales.

d) Institucionales: Favorecer el acceso al sistema federativo, mejorar el acceso a instalaciones públicas, modificar la legislación deportiva.

Tales demandas no están muy alejadas de las que plantea la población barcelonesa en general. De acuerdo con la encuesta de hábitos deportivos de los barceloneses, la mayoría de la población demanda una mejora de los equipamientos públicos urbanos, gratuitos y abiertos a toda la población (Ajuntament de Barcelona 2006b). 


\section{CRITERIOS PARA LA PROMOCIÓN DEPORTIVA CIUDADANA}

Es habitual, al hablar de deporte e inmigración, centrar el debate sobre la interculturalidad. Son varios los autores (Kennett 2005; Lleixà 2002; Lleixà y Soler 2004; Bantulà y Mora 2002) que trabajan con una serie de conceptos ya habituales en estos debates: multiculturalismo, policulturalismo e interculturalismo. Básicamente, se habla de multiculturalismo cuando gente con culturas distintas comparte el mismo territorio, cosa que podría lleva a la interacción. Se emplea pluriculturalismo cuando existen diferentes culturas que conviven en un mismo territorio pero que no interactúan, e interculturalismo cuando se comparte e interactúa con gentes de culturas diferentes a la propia, encontrando que "puede haber una mezcla de pluriculturalismo y interculturalismo en un contexto multicultural" (Kennett 2005: 9).

Sin embargo, en nuestra opinión todas estás aproximaciones tienen como elemento central un concepto de cultura erróneo. Una conceptualización de la cultura tratada como esencial, ideal-culturalista, homogénea y totalizadora. Un concepto de cultura que hace de la diferencia algo exterior y anula el moderno proceso de diferenciación interna que evoluciona por una doble diferenciación: funcional (subsistemas sociales, campos, etc.) y social (estamentos, clases, agrupaciones, individuo, etc.) (Luhmann 1998). Desde una perspectiva más acertada, la cultura debe ser vista como un procesoflujo siempre inestable que acompaña la complejización de los sistemas sociales y la tendencia a la "individualización institucionalizada" (Beck 2001); una "Unidad Múltiple" con creciente complejidad (Beriain 1996).

En esta línea se manifestaba Delgado cuando criticaba por racista la idea del multiculturalismo como una convivencia en mosaico de grupos que no se interseccionan, de culturas singulares planteadas como universos herméticos e inconmensurables. En definitiva,

els que defensen el mestissatge cultural, en la mesura que proclamen que les cultures s'han de mesclar estan suggerint que poden no fer-ho. Davant d'aquesta premisa s'ha de proclamar que no cal barrejar les cultures, perqué de fet no poden fer altre cosa, i cadascuna d'elles és en si mateixa una barreja (Delgado 1998: 127).

En efecto, es más lícito hablar de transculturalidad, de hibridación cultural y de procesos de bricolaje personal con la cultura. Medina señalaba la necesidad de acercarse a la interculturalidad y el deporte desde una perspectiva voluntarista que huyera de un concepto de integración esencialista y que reconociera la necesidad de "una renegociación continua [...] entre las diversas partes que componen el entramado social" (Medina 2002: 22). En esta idea abunda la propuesta conceptual de Beck cuando afirma que el cos- 
mopolitismo metodológico se debe imponer frente a la creciente contradicción existente entre el nacionalismo metodológico y la cosmopolitización real: "La mirada nacional, la gramática nacional, es falsa porque la acción política, económica y cultural, junto con sus consecuencias, conscientes e inconscientes, no pueden conocer fronteras en la sociedad del riesgo transnacional" (Beck 2005: 37).

A partir del nacionalismo metodológico se construye una definición implícita de lo que es o no es una persona inmigrante y se conforma un sistema de diferenciación con respecto a los individuos autóctonos. Esta elaboración se hace a través de dos elementos clave en el discurso (Tello et al. 2008): el nuevo referente de la autoctonía que necesita de la alteridad para la construcción identitaria de un nosotros (la sociedad de acogida), y una representación estereotipada de la inmigración, especialmente en la representación de la mujer inmigrada. Un estereotipo que se construye en todo momento desde el modelo de domesticidad y el subdesarrollo. La integración se plantea como un pacto no negociado, donde la sociedad de acogida establece las políticas de integración y se representa como agente activo, mientras se muestra a la inmigración como agente pasivo y, por ello, no se le reconoce el derecho a modificar las condiciones del pacto. Existen supuestos derechos inalterables de la sociedad de acogida que están por encima de los procesos democráticos. En general, se olvidan las aportaciones positivas de la inmigración y se destacan las negativas (Tello et al. 2008).

Se trata de lo que Beck denomina "la doble cara del universalismo": la presión por los valores humanos universales, normalmente ligada a lo propio, puede llegar a diluir la alteridad, especialmente la de los otros. "A la postulada igualdad abstracta corresponde la presión sobre el étnicamente distinto a plegarse a esta pretensión de igualdad particular, es decir, a renunciar a la posición de la diferencia" (Beck 2005: 77). En efecto, la integración es el problema de las sociedades contemporáneas, en todos sus sentidos: simbólica, funcional, moral, social. Se trata, en definitiva de una integración laxa y no recurrente. Mientras que, sin embargo, la exclusión social se muestra totalmente integrada, llega a todos los sistemas y es recurrente. (Luhmann 1998). Algo similar plantea Heinemann (2002) en relación a la integración social y el deporte. El autor sugiere diferentes campos para la integración social: legal, estructural-funcional, instrumental, cultural, identitario, donde la integración en uno de los campos, no garantiza, en absoluto, la integración total. Por ello, la integración deportiva no garantiza la integración social, pero la exclusión legal sí es recurrente y redundante en todos los sistemas, también en el deportivo.

Por otra parte, el deporte es un sistema productor de sentido en las sociedades modernas. Convertido en un elemento clave de comunicación 
entre sistemas sociales y sistemas psíquicos, y medio de generalización simbólica, funciona como un instrumento de fusión, un bálsamo social que ofrece una reducción de la contingencia simbólica, redundancia discursiva y seguridad ontológica (Sánchez 2003). Es aquí donde el deporte, como antropología de la igualdad de los individuos, armoniza simbólicamente las contradicciones culturales señaladas por Bell (1987) y se convierte en una metanarrativa práctica cargada de sentido. Especialmente el deporte urbano permite hacer de los espacios públicos zonas de contactos que configuran formas culturales híbridas y se convierten en referentes simbólicos e identitarios (Tello et al. 2008). En el caso específico de Barcelona, diferentes estudios sobre el deporte en los espacios públicos ponen, además, de relieve su papel en la formación de redes sociales ${ }^{6}$ y su importancia en el desarrollo del capital social. En este sentido se manifiesta Maza (2006: 208) cuando afirma que "el capital social de las actividades deportivas [...] es un capital para las relaciones sociales [...]. Para el "bricolaje social"”.

A partir de todo lo anterior, podemos formular un avance de discusión acerca de los principios básicos sobre los que elaborar un programa de promoción de la práctica deportiva de la ciudadanía ${ }^{7}$.

a) Principio de igualdad. Equiparación de derechos, deberes y oportunidades de los ciudadanos:

Promoción de la igualdad de oportunidades y en contra de la exclusión social.

Apuesta por actuaciones encaminadas al logro de porcentajes de participación deportiva similares entre nacionales de terceros países y autóctonos.

Fomento de la práctica deportiva para personas en situación de especial vulnerabilidad.

Introducción de la perspectiva de género.

b) Perdurabilidad. Acción sostenida en el tiempo hasta la obtención de resultados.

c) Integralidad. Tratamiento integral del conjunto de ámbitos que pueden afectar la integración de los ciudadanos a través de un enfoque holístico del fenómeno migratorio: Equiparación en cuanto a trato de las políticas deportivas en relación a otras políticas sectoriales (trabajo, educación, vivienda, sanidad,...).

\footnotetext{
${ }^{6}$ Estas investigaciones se encuentran reunidas en el monográfico de la revista Apunts. Educació Física y deportes, n. 91, que ha sido coordinado por Núria Puig y Gaspar Maza (2008).

7 Para conocer el marco teórico que sustenta estos principios y criterios véase Bantulà y Sánchez (2008).
} 
Apuesta decidida por un tratamiento transversal que se traduzca en la inclusión del deporte en los planes de inmigración, en las políticas de integración y en las políticas de promoción deportiva de las diferentes administraciones.

Enfoque holístico del fenómeno migratorio.

d) Normalización. Inclusión de los inmigrantes en los sistemas generales de servicios que el Estado del bienestar presta a la ciudadanía: Aprovechamiento de los servicios deportivos y sociales existentes, si bien reorientados para poder atender a la diversidad de ciudadanos, así como ampliación de los recursos para afrontar el aumento de población que hace uso de los servicios.

Adecuación de la oferta deportiva a las demandas de una sociedad diversa.

e) Participación. Presencia de los diferentes agentes y organizaciones sociales en el proceso de elaboración, así como durante la ejecución y evaluación del plan:

Implicación del tercer sector en el diseño y aplicación de las políticas de promoción deportiva.

Fomento del asociacionismo y de la participación de las agrupaciones y entidades de inmigrantes con respecto a la incorporación de la práctica de actividades físico-deportivas.

Implicación de entidades, clubes, federaciones y asociaciones deportivas.

f) Coordinación. Establecimiento de tareas de coordinación entre las diferentes administraciones locales, autonómicas y estatales:

Creación de mecanismos que promuevan la coordinación de técnicos que intervienen en materia deportiva dentro de la Administración local.

Incentivación de la coordinación de diferentes ámbitos de la Administración local.

Impulso de la coordinación con el resto de administraciones.

Contactos periódicos entre Administración y el sector asociativo.

Optimización y racionalización de los recursos de los diferentes agentes públicos y privados.

Despliegue de trabajo en red entre los agentes políticos, sociales y económicos.

g) Descentralización. El plan se adecua al territorio con la participación de las administraciones locales y de las organizaciones sociales en la ejecución de las políticas públicas:

Adecuación de la promoción deportiva como ámbito sectorial a su territorialidad. 
Aplicación coherente de los mismos criterios de promoción deportiva, pero con programas, intensidad y ritmos diferentes según la realidad de cada barrio y distrito de la ciudad.

h) Cooperación. Fomentar políticas y experiencias de co-desarrollo con los países de origen de la inmigración:

Apoyo a iniciativas de co-desarrollo en el ámbito deportivo.

i) Transculturalidad. Estimular las relaciones recíprocas entre todos los grupos de ciudadanos, favoreciendo los procesos de hibridación cultural personal:

Valoración y respeto de la diversidad de culturas deportivas.

Reconocimiento de la transculturalidad y de la hibridación cultural y personal. Biografías de bricolaje.

j) Financiación. Dotación presupuestaria en cada una de las acciones del plan que lo requieran:

Determinación de fórmulas para conseguir vías de financiación.

Búsqueda de la co-financiación.

Despliegue de dotación presupuestaria en cada una de las acciones del plan que lo requieran.

\section{CONCLUSIONES}

Concluiremos con dos líneas de reflexión:

Por una parte, aquella que hace referencia a la práctica deportiva. No hay duda de la multicausalidad que condiciona el desarrollo de la práctica deportiva. El origen étnico y las diversas culturas corporales y deportivas asociadas no son determinantes ni inmutables. Puesto que los diacríticos básicos son los mismos que para toda la ciudadanía, hay que seguir mejorando las condiciones sociales que hacen posible su práctica. No se destacan lo suficiente los aspectos positivos que conlleva para el sistema deportivo la llegada de población inmigrante. En efecto, su inserción en el sistema deportivo federativo ha incrementado de un 10 a un $30 \%$ las licencias en deportes como el béisbol, el ajedrez, el voleibol, o la lucha. También ha permitido la aparición en nuestro país de otras prácticas como el criquet (con una propuesta de federación catalana). Permite, así, un renovado Sistema Deportivo Híbrido que suma las aportaciones deportivas de todos los ciudadanos. Hay que hacer notar también, que se ha producido la institucionalización del binomio deporte-integración social, pero exclusivamente en referencia a aquellos colectivos considerados en falta permanente de integración: inmigrantes, colectivos en riesgo social, etc. Por último, hay que abundar en programas para la promoción del deporte en relación a la salud. 
En segundo lugar, por lo que respecta a las reflexiones teóricas y metodológicas, creemos que los estudios sobre deporte e inmigración permiten poner en discusión las formas: Inmigrante-Autóctono; Sociedad de acogida-Nuevos ciudadanos, como construcciones socioculturales a denunciar. La actividad físicodeportiva permite ejemplificar el debate sobre la transculturalidad y su incardinación en biografías de bricolaje: individuos políglotas, cosmopolitas con múltiples identificaciones. Por último, queremos hacer referencia a la importancia de utilizar una epistemología alejada de los parámetros tradicionales que tratan lo nacional vinculado a un territorio, una sociedad y una cultura, para acercarnos a planteamientos como el del cosmopolitismo metodológico planteado por Beck (2005) donde los planteamientos identitarios nunca son excluyentes.

\section{BIBLIOGRAFÍA CITADA}

Ajuntament de Barcelona. 2002. Drets i deures dels immigrants/Derechos y deberes de los inmigrantes (varios idiomas). Barcelona: Ajuntament de Barcelona.

Ajuntament de Barcelona. 2003a. Pla Estratègic de l'Esport de Barcelona. Barcelona: Ajuntament de Barcelona.

Ajuntament de Barcelona. 2003b. Pla Municipal d'Immigració. Barcelona: Ajuntament de Barcelona.

Ajuntament de Barcelona. 2006a. Informes Estadístics. La població estrangera a Barcelona. Barcelona: Ajuntament de Barcelona.

Ajuntament de Barcelona. 2006b. Enquesta. Hàbits Esportius a Barcelona. Presentació de resultats. Barcelona: Ajuntament de Barcelona.

Ajuntament de Barcelona. 2007. Estudi dels hàbits esportius de la població en edat escolar en funció del gènere i del tipus de pràctica a la ciutat de Barcelona. Barcelona: IBE.

Alberich, T. 2000. "Perspectivas de la investigación social", en T. R. Villasante et al., Investigación social participativa. Construyendo ciudadania/1: 59-72. Barcelona: El Viejo Topo.

Balibrea, E. y A. Santos. 2006. "El deporte como medio de inserción de mujeres en contextos socialmente desfavorecidos", en X. Pujades et al. (comps.), Culturas deportivas y valores sociales: 61-69. Madrid: Librerías Deportivas Esteban Sanz.

Bantulà, J. y J. Mora. 2002. Juegos multiculturales: 225 juegos tradicionales para un mundo global. Barcelona: Paidotribo.

Bantulà, J. y R. Sánchez. 2008. "Inmigración y culturas deportivas: Estudio para determinar los criterios de intervención y promoción deportiva municipal en Barcelona", en L. Cantarero, X. Medina y R. Sánchez (comps.), Actualidad en el deporte: investigación y aplicación: 121-137. Donostia: Ankulegui.

Bantulà, J. y R. Sánchez. 2009. "Deporte e inmigración en Barcelona: criterios para una promoción deportiva ciudadana" en A. Vilanova, et al. (comps.), Deporte, Salud y Medioambiente: 345-355. Madrid: AEISAD-Librerías Deportivas Esteban Sanz.

Basagoiti, M. у P. Bru. 2000. "Mira quién habla. El trabajo con grupos en la IAP", en T. Villasante; M. Montañés y P. Martín (coords.), Investigación social participativa. Construyendo ciudadania/1: 119-136. Barcelona: El Viejo Topo. 
Bauman, Z. 2000. Modernidad Liquida. Buenos Aires: FCE.

Bauman, Z. 2002. La cultura como praxis. Barcelona: Paidós.

Beck, U. 1998. La sociedad del riesgo. Barcelona: Paidós.

Beck, U. 2001. "Vivir nuestra propia vida en un mundo desbocado. Individualización, globalización y política", en A. Giddens y W. Hutton (eds.), En el limite. La vida en el capitalismo global: 233-246. Barcelona: Tusquets.

Beck, U. 2005. La mirada cosmopolita o la guerra es la paz. Barcelona: Paidós.

Bell, D. 1987. Las contradicciones culturales del capitalismo tardio. Madrid: Alianza.

Beriain, J. 1996. La integración en las sociedades modernas. Barcelona: Anthropos.

Bourdieu, P. 1997, Razones prácticas. Sobre la teoría de la acción. Barcelona: Anagrama.

Brunet, I.; I. Pastor y À. Belzunegui. 2002. Tècniques d'investigaciò social. Fonaments epistemològics i metodològics. Barcelona: Pòrtic.

Buxó, M. J. 2002. "La conjunció aplicada dels 'co-': comitès, col-laboracions, col-legues, co-operacions, co-generació i co-gestió de dades i problemas". Revista d'Etnologia de Catalunya 20: 130-141.

Delgado, M. 1998. Diversitat i integració. Barcelona: Empúries.

Domínguez, M. y A. Coco. 2000. "El pluralismo metodològic com a posicionament de partida. Una primera valoració del seu ús a la recerca social". Revista Catalana de Sociología. 11: 105-132.

Durán, J. 2002. "Inmigración y actividad físico-deportiva", en II Congreso de Ciencias del Deporte. Vol. I: 179-200. Madrid: INEF.

Gabarrón L. R. y L. Hernández. 1994. Investigación Participativa. Madrid: CIS.

Heinemann, K. 2002. "Esport per a immigrants: instrument d'integració?", en N. Puig (coord.), Dossier Esport i Immigració, Apunts. Educació Física i Esports n.ำ 68: 24-35. Barcelona: INEFC.

Kennett, C. 2005. Esport, immigració i multiculturalitat: una anàlisi conceptual. Barcelona: Centre d'Estudis Olímpics UAB.

Luhmann, N. 1998. Complejidad y modernidad. De la unidad a la diferencia. Madrid: Trotta.

Lleixà, T. y S. Soler. 2004. Actividad física y deporte en sociedades multiculturales. Barcelona: Paidotribo.

Lleixà, T. et. al. 2002. Multiculturalismo y educación física. Barcelona: Paidotribo.

Maffesoli, M. 1997. Elogio de la razón sensible. Barcelona: Paidós.

Martín, P. 2001. "Balance de diferentes técnicas participativas para integrar metodologías creativas", en T. Villasante, M. Montañés y P. Martín (coords.). 2001. Prácticas locales de creatividad social. Construyendo ciudadania/2: 41-66. Barcelona: El viejo Topo - Red CIMS.

Maza, G. 2002. "L'esport i el seu paper en els mecanismos de reproducció social", en N. Puig (coord.), Dossier Esport i Immigració, Apunts. Educació Física i Esports. n.. 68: 58-67. Barcelona: INEFC.

Maza, G. 2006. "El capital social del deporte", en X. Pujadas, et. al. (comps.), Culturas deportivas y valores sociales: 199-210. Madrid: Librerías Deportivas Esteban Sanz.

Medina, F. X. 2002. "Esport, immigració i interculturalitat", en N. Puig (coord.), Dossier Esport i Immigració, Apunts. Educació Física i Esports. n.o 68: 18-23. Barcelona: INEFC.

Medina, F. X. y R. Sánchez (eds.). 2003. Culturas en juego. Ensayos de antropología del deporte en España. Barcelona: Icaria/ICA.

Outhwaite, W. 2008, El futuro de la sociedad. Madrid: Amorrortu Editores. 
Puig, N. (coord.). 2002. Dossier Esport i Immigració, Apunts. Educació Física i Esports. no 68. Barcelona: INEFC.

Puig, N. y G. Maza (coords.). 2008. Dossier "El deporte en los espacios públicos urbanos". Apunts. Educació Física i Esports 91.

Rodríguez, J. M. 2006. "Posibilidades y límites del deporte en el ámbito de la exclusión social”, en X. Pujadas, et al. (comps.), Culturas deportivas y valores sociales: 257-264. Madrid: Librerías Deportivas Esteban Sanz, S. L.

Roigé, X. et al. 1999. Têcniques d'investigació en antropologia social. Barcelona: Edicions de la Universitat de Barcelona.

Sánchez, R. 2003. "El deporte ¿nuevo instrumento de cohesión social?", en F. X. Medina y R. Sánchez (eds.), Culturas en juego. Ensayos de antropología del deporte en Espa$\tilde{n} a$ : 49-72. Barcelona: Icaria/ICA.

Sánchez, R. 2004. "Nuevos usos de la ciudad: actividades lúdico-deportivas y

apropiación del espacio urbano, en C. Ortiz (ed.), La ciudad es para ti. Nuevas y

viejas tradiciones en ámbitos urbanos: 165-186. Barcelona: Anthropos.

Sánchez, R. 2009. Deporte, socialidad y sentido en la sociedad contemporánea. Milán: Università Cattolica del Sacro Cuore.

Secretaria per a la Inmigració. 2006. Pla de ciutadania i immigració 2005-2008. Barcelona: Generalitat de Catalunya.

Tello, R., N. Benach y M. Nash (eds.). 2008. Intersticios. Contactos interculturales, género y dinámicas identitarias en Barcelona. Barcelona: Ediciones Bellaterra.

Villasante, T.; M. Montañés y P. Martín (coords.). 2000. La investigación social participativa. Construyendo ciudadania/1. Barcelona: El Viejo Topo.

Villasante, T.; M. Montañés y P. Martín (coords.). 2001. Prácticas locales de creatividad social. Construyendo ciudadania/2. Barcelona: El viejo Topo - Red CIMS.

Recibido: 6 de abril de 2010.

Aprobado: 22 de junio de 2010. 